\title{
Comparison of the Combined Anterior-Posterior Approach versus Posterior-Only Approach in Scoliosis Treatment
}

\author{
Hossein Hojjat Pourfeizi ${ }^{1}$, Jafar Ganjpour Sales ${ }^{1}$, Ali Tabrizi ${ }^{1}$, Ghanbar Borran ${ }^{2}$, Sahar Alavi ${ }^{1}$ \\ ${ }^{1}$ Department of Orthopedic Surgery, Shohada Educational Hospital, Tabriz University of Medical Sciences, Tabriz, Iran \\ ${ }^{2}$ Department of Neurosurgery, Shohada Educational Hospital, Tabriz University of Medical Sciences, Tabriz, Iran
}

\begin{abstract}
Study Design: This is descriptive analytical study.
Purpose: The present study aims at comparing treatment results found between the two groups comprising of patients who underwent posterior spinal fusion using thoracic pedicle screws and the ones who underwent combined anterior-posterior method, respectively.

Overview of Literature: There was controversy about surgical techniques including anterior, posterior, or a combined anteriorposterior approaches are applied to treat non-congenital scoliosis with surgical indications.

Methods: Medical records of 50 patients suffering from thoracic non-congenital scoliosis with curves exceeding $70^{\circ}$ were reviewed. In this study, 25 patients who underwent posterior spinal fusion using thoracic pedicle screws were compared with 25 patients who underwent combined anterior-posterior method.

Results: Patients treated through posterior-only and combined approaches were respectively hospitalized for $11.84 \pm 5.18$ and $26.5 \pm 5.2$ days $(p=0.001)$. There was a significant difference between these two groups considering intensive care unit admission duration ( $p=0.001)$, correction in sagittal view of $X$-ray $(p=0.01)$, and number of days the patients underwent traction $(0.001)$. Finally, coronal view was corrected without any significant difference $(p=0.2)$.

Conclusions: According to our findings, it is hypothesized that posterior-only method is associated with some significant advantages and is an advisable method in patients with severe scoliosis over than $70^{\circ}$.
\end{abstract}

Keywords: Scoliosis; Curved spine; Spinal deformity

\section{Introduction}

Scoliosis (meaning "crooked") is a medical condition where a person's spine is curved from side to side [1]. It is the most common type of spinal deformity confronted by the orthopedic surgeons [2]. A scoliosis spinal columns curve $\leq 10^{\circ}$ affects $1.5 \%$ to $3 \%$ of people [3]. Prevalence of curves $<20^{\circ}$ is almost equal in males and females. It is most common during late childhood [4]. The condition affects approximately 6 to 7 million people in the United States. Considering the most common form of scoliosis, adolescent idiopathic scoliosis (AIS), there is no clear causal factor and it is generally believed to be a multifactor problem [5]. However, a primary muscle disorder

Received Sep 24, 2012; Revised Oct 29, 2012; Accepted Oct 31, 2012

Corresponding author: Ali Tabrizi

Department of Orthopedics Surgery, Shohada Educational Hospital,

El Goli St., Center of Tabriz University of Medical Sciences, Tabriz, Iran

Tel: +98-9148883851, Fax: +98-4113859314, E-mail: Tabrizia@Tbzmed.ac.ir 
Table 1. Demographic characteristics and other comparison points of the patients in both groups

\begin{tabular}{lccc} 
Variable & Posterior-only approach $(\mathrm{n}=25)$ & Anterior-posterior (AP) approach $(\mathrm{n}=25)$ & $p$-value \\
\hline Sex (male/female) & $14 / 11$ & $12 / 13$ & 0.5 \\
\hline Age (yr) & $18.2 \pm 5.5$ & $19.6 \pm 7.2$ & 0.5 \\
\hline Hospitalized days & $11.84 \pm 5.1$ & $26.52 \pm 5.21$ & $<0.001$ \\
\hline Intensive care unit stay (day) & $1.52 \pm 0.6$ & $4.68 \pm 3.8$ & $<0.001$ \\
\hline Traction duration (day) & $1.68 \pm 3.9$ & $10.52 \pm 5$ & $<0.001$ \\
\hline Correction degree (AP view) & $41.2 \pm 12.8$ & $45.4 \pm 12.4$ & 0.2 \\
\hline Correction degree (lateral view) & $12.04 \pm 15$ & $23.2 \pm 17.04$ & 0.01 \\
\hline
\end{tabular}

and/or genetic factors are believed to be the responsible etiologies. AIS is the most common type of idiopathic scoliosis and the most common type of scoliosis overall [5].

The traditional medical management of scoliosis is complex. The conventional options sequentially include observation, bracing, and surgery. Spinal deformity is surgically operated to correct or improve deformity: to maintain sagittal balance, to preserve or to improve pulmonary function, to minimize morbidity or pain, to maximize postoperative function, to improve or at least not to harm the function of the lumbar spine [6]. Indications for operative treatment of AIS include developed curvature in the growing children, severe deformity $\mathrm{C}>50$ with asymmetry of trunk in adolescents, pain which cannot be controlled by non-operative treatments, thoracic lordosis, and significant physical deformity. Surgical techniques may include anterior, posterior, or combined anteriorposterior fusion surgery [6,7]. Anterior techniques include anterior instrumentation and fusion for idiopathic scoliosis which is now regarded as a well-accepted procedure for certain thoracolumbar and lumbar curves [6,7]. Posterior techniques include posterior fusion and instrumentation by pedicle screws or hooks without using the anterior approach. Combined techniques include two stages: 1) anterior release and fusion and 2) posterior fusion and instrumentation using multi-hook segments [6,7]. A combination of these two stages is used in more severe cases. Scoliosis has been surgically treated for the past 15 years at Tabriz Shohada Hospital. This study aims at comparing two surgical methods, combined anteriorposterior approach using hybrid lumbar pedicle screws and thoracic hooks with posterior-only approach using lumbar and thoracic pedicle screw systems to treat severe scoliosis with curvature $>70^{\circ}$.

\section{Materials and Methods}

Medical records of 50 patients suffering from thoracic non-congenital scoliosis with curves exceeding $70^{\circ}$ were reviewed. The case group consisted of 25 patients who underwent posterior spinal fusion using thoracic pedicle screws (group A) and the control group consisted of other 25 patients (group B), who underwent anterior release by thoracotomy approach in the first stage and postoperative traction for 8 to 10 days, respectively. Traction included distal femoral skeletal and skull tractions. At the second stage, the patients underwent posterior fusion and instrumentation using pedicle screws in lumbar vertebra and pedicle hooks and transverse hook to upper thoracic. Considering radiography, all curves of the thoracic, thoracolumbar, and lumbar spine were measured using coronal Cobb measurement.

Patients with congenital scoliosis and anomalies of spinal canal were excluded from the study. The two groups were compared considering correction rate, days of hospitalization, duration of traction, total cost of treatment, and postoperative complications. Written informed consent was obtained from all patients. The study was approved by Ethics Committee of Tabriz University of Medical Sciences.

\section{Statistical analysis}

The statistical analysis of data was carried out using the SPSS ver. 16 (SPSS Inc., Chicago, IL, USA). Continuous variables are shown as mean \pm standard deviation. Fisher's exact test or chi-square test was used to study qualitative variables. Independent $t$-test was used to evaluate the quantitative variables between the two groups. In this study, $p \leq 0.05$ was regarded as statistically significant. 


\section{Results}

The present study was conducted on fifty patients. Demographic characteristics of patients of both the groups are shown in Table 1. No significant difference was observed between the two groups with respect to gender and age.

There was significant difference between the two groups considering duration of hospitalization, intensive care unit (ICU), traction, and correction degree seen in lateral view (Table 1). Figs. 1 and 2 refer to radiographic and clinical images of one patient treated using posterioronly method and a combined posterior-anterior approach, respectively.
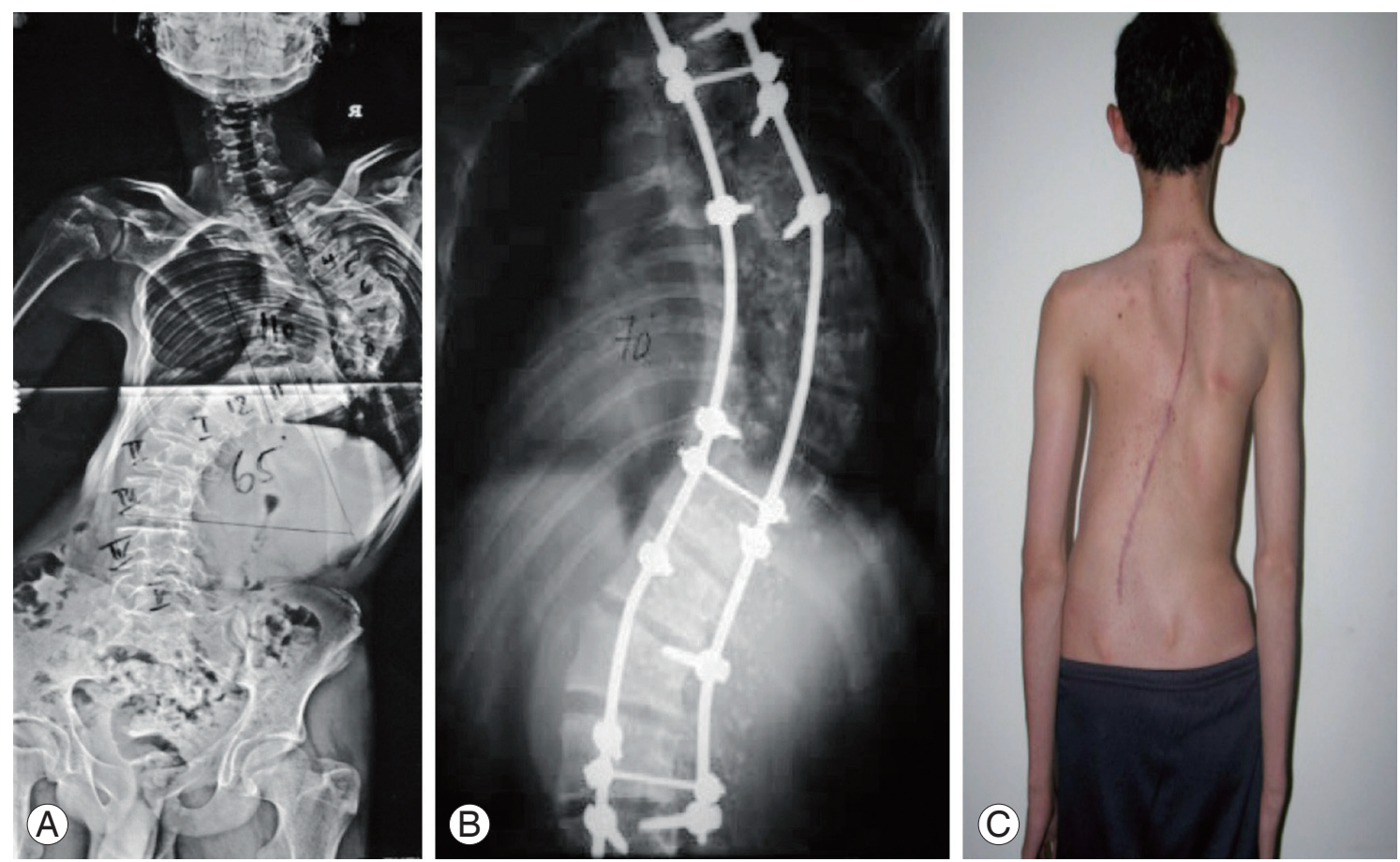

Fig. 1. Preoperation (A), postoperation (B) X-ray graphs and three-year follow-up (C) images of a subject of posterior-only approach group.
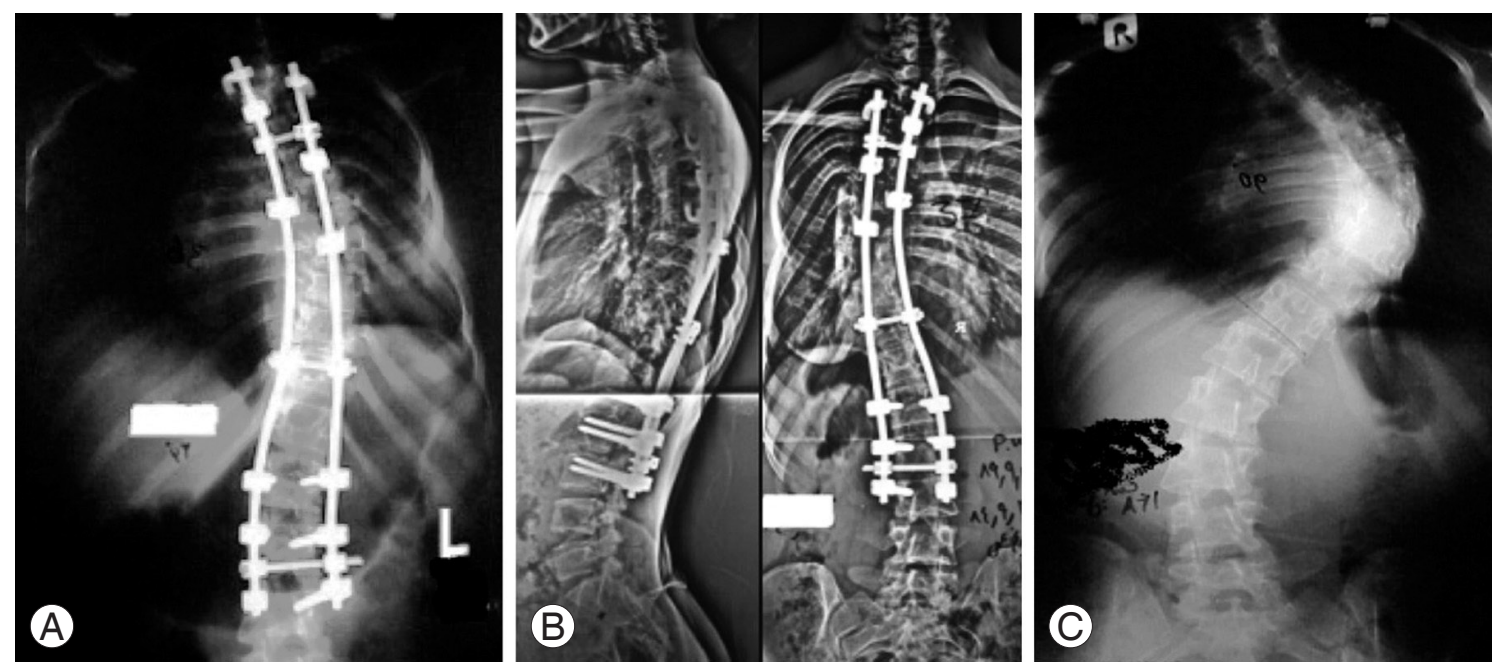

Fig. 2. Preoperation (A), postoperation (B), and five-year follow-up (C) X-ray graphs of a subject of anterior-posterior approach group. 


\section{Discussion}

Rate of correction is one of the most important goals of all the surgical methods [7]. There are various reports in the literature comparing anterior releasing and posterior fusion methods in treating scoliosis [7]. Yamin et al. [8] published a retrospective study on a staged surgery for severe rigid scoliosis with coronal Cobb angle $>80^{\circ}$ treated by staged surgeries including anterior release and halo-pelvic traction as the first and posterior instrumentation and spinal fusion as the second stage. They concluded that staged operation offers a safe and effective way in treating severe rigid scoliosis [8]. Min et al. [9] assessed clinical, radiological and outcomes of the patients suffering from idiopathic AIS and its treatment by selective short anterior fusion of the major thoracolumbar/ lumbar (TL/L) curve. They concluded that selective short anterior fusion of the TL/L curve scoliosis with a thoracic curve $\leq 25^{\circ}$ (according to Lenke classification, type V) results in a satisfactory corrected and a balanced spine. Short fusions leave enough mobile lumbar segments for establishment of global spinal balance [9].

Considering correction rate of the curves, treatment of AIS $>80^{\circ}$ using posterior-only fusion method is comparable to most of the series of combined anterior-posterior surgery with shorter operation time without leaving any adverse effect on pulmonary function of anterior transthoracic exposure $[6,9]$. Compared with hybrid construct, posterior spinal fusion method used by only pedicle screws in severe cases of thoracic AIS allowed a greater coronal correction of both main thoracic and secondary lumbar curves, and resulted in less loss of the postoperative correction achieved, and fewer revision surgeries were required. Using posterior-only fusion method with pedicle screws enabled a good and stable correction of severe scoliosis. However, severe curves may be amenable to hybrid instrumentation, which has produced results similar to those of the screw-only construct with regards to patient satisfaction [10].

Suk et al. [11] compared segmental pedicle screw fixation method with multi-hook segmental fixation systems in treating thoracic idiopathic scoliosis. The correction rate was estimated as 55\% and $72 \%$ using hooks and segmental thoracic pedicle screws, respectively. The study reported a $3 \%$ malposition rate for thoracic pedicle screws based on plain radiographs. There was neither medial intracanal malposition nor any neurological complica- tions [11]. Luhmann et al. [12] found that posterior-only thoracic pedicle screw constructs provided correction rate equal to that of hook constructs with anterior release.

Thoracic pedicle screw instrumentation, therefore, eliminates need for an anterior approach. Sanders et al. [13] found significantly better major and minor curvature correction rates without any neurological problem and improved pulmonary function with posterior pedicle screw instrumentation as compared with segmental hook instrumentation. Betz et al. [14] reported that coronal correction and balance were equal in both anterior and posterior groups. In the anterior group there was a better correction of sagittal profile in patients with a preoperative hypokyphosis $<20^{\circ}$. However, hyperkyphosis (with a mean of $54^{\circ}$ ) occurred in $40 \%$ of members of the anterior group with a preoperative kyphosis $>20^{\circ}$ [14].

Thoracic pedicle screws improved correction in both coronal and axial planes. Lumbar lordosis can be satisfactorily controlled to produce more thoracic hypokyphosis in comparison with posterior segmental hook instrumentation.

However, sagittal correction of scoliosis was significantly different between the two groups but there was no statistically significant difference with respect to coronal correction. Bi-step surgery to correct scoliosis may result in some complications since wound or anesthesia-related complications might be at a risk of increase. Complications of thoracotomy (haemothorax, pneumothorax, etc.) are other potential threats. Postoperative events should also be noted. Because of special complications of ICU admissions, statistically significant difference is an important factor considering ICU admission. Total hospitalization duration is another factor that should be mentioned. Long-term hospitalization may result in increase in risks related to hospitalization (medical errors, nosocomial infections, psychological effects, etc.) [15].

The risk of scoliosis surgery is estimated to be $5 \%$. Inflammation of the soft tissue or deep inflammatory processes, breathing impairments, bleeding, and nerve injuries may be regarded as possible complications. As early as five years after surgery, about $5 \%$ of the patients require reoperation [16]. The most common form of scoliosis never exceeds $80^{\circ}$ [16]. Unfortunately, the physical effects of surgery are not necessarily stable [5]. Posterior segmental pedicle screw fixation without anterior release had resulted in satisfactory deformity correction in severe scoliosis without significant loss of curvature correction. 
In this series, a single posterior procedure obviated the need for the anterior release and eliminated complications related to anterior surgery [13].

\section{Conclusions}

According to our findings, it is hypothesized that posterior-only method is associated with some significant advantages and is an advisable method in patients with severe scoliosis over than $70^{\circ}$.

\section{Conflict of Interest}

No potential conflict of interest relevant to this article was reported.

\section{Acknowledgments}

This study was financially supported by Tabriz University of Medical Sciences.

\section{References}

1. Goldberg CJ, Moore DP, Fogarty EE, Dowling FE. Scoliosis: a review. Pediatr Surg Int 2008;24:129-44.

2. Lonstein JE, Winter RB, Bradford DS, Ogilvie JW. Moe's textbook of scoliosis and other spinal deformities. 3rd ed. Philadelphia: Saunders; 1995.

3. Herring JA. Tachdjian's pediatric orthopaedics. 4th ed. Philadelphia: Saunders Elsevier; 2002.

4. Marieb EN. Human anatomy and physiology. San Francisco: Benjamin Cummings; 1998.

5. Kouwenhoven JW, Castelein RM. The pathogenesis of adolescent idiopathic scoliosis: review of the literature. Spine (Phila Pa 1976) 2008;33:2898-908.

6. Canale ST, Beaty JH. Campbell's operative orthopaedics. 11th ed. Philadelphia: Mosby; 2008.

7. Muschik MT, Kimmich H, Demmel T. Comparison of anterior and posterior double-rod instrumenta- tion for thoracic idiopathic scoliosis: results of 141 patients. Eur Spine J 2006;15:1128-38.

8. Yamin S, Li L, Xing W, Tianjun G, Yupeng Z. Staged surgical treatment for severe and rigid scoliosis. J Orthop Surg Res 2008;3:26.

9. Min K, Hahn F, Ziebarth K. Short anterior correction of the thoracolumbar/lumbar curve in King 1 idiopathic scoliosis: the behaviour of the instrumented and non-instrumented curves and the trunk balance. Eur Spine J 2007;16:65-72.

10. Di Silvestre M, Bakaloudis G, Lolli F, Vommaro F, Martikos K, Parisini P. Posterior fusion only for thoracic adolescent idiopathic scoliosis of more than 80 degrees: pedicle screws versus hybrid instrumentation. Eur Spine J 2008;17:1336-49.

11. Suk SI, Kim JH, Cho KJ, Kim SS, Lee JJ, Han YT. Is anterior release necessary in severe scoliosis treated by posterior segmental pedicle screw fixation? Eur Spine J 2007;16:1359-65.

12. Luhmann SJ, Lenke LG, Bridwell KH, Schootman M. Revision surgery after primary spine fusion for idiopathic scoliosis. Spine (Phila Pa 1976) 2009;34:21917.

13. Sanders AE, Baumann R, Brown H, Johnston CE 2nd, Lenke LG, Sink E. Selective anterior fusion of thoracolumbar/lumbar curves in adolescents: when can the associated thoracic curve be left unfused? Spine (Phila Pa 1976) 2003;28:706-13.

14. Betz RR, Harms J, Clements DH 3rd, et al. Comparison of anterior and posterior instrumentation for correction of adolescent thoracic idiopathic scoliosis. Spine (Phila Pa 1976) 1999;24:225-39.

15. Weiss HR, Goodall D. Rate of complications in scoliosis surgery: a systematic review of the Pub Med literature. Scoliosis 2008;3:9.

16. Hawes MC, O'Brien JP. A century of spine surgery: what can patients expect? Disabil Rehabil 2008;30:808-17. 